\title{
Fabrication and Deterministic Transfer of High-Quality Quantum Emitters in Hexagonal Boron Nitride
}

\author{
Tobias Vogl,*,† Geoff Campbell, ${ }^{\dagger}$ Ben C. Buchler, ${ }^{\dagger}$ Yuerui Lu, ${ }^{\ddagger}$ a and Ping Koy Lam* ${ }^{\dagger}$ \\ ${ }^{\dagger}$ Centre for Quantum Computation and Communication Technology, Department of Quantum Science, Research School of Physics \\ and Engineering, The Australian National University, Acton, ACT 2601, Australia \\ ${ }^{\ddagger}$ Research School of Engineering, The Australian National University, Acton, ACT 2601, Australia
}

Supporting Information

\begin{abstract}
Color centers in solid state crystals have become a frequently used system for single-photon generation, advancing the development of integrated photonic devices for quantum optics and quantum communication applications. In particular, defects hosted by two-dimensional (2D) hexagonal boron nitride (hBN) are a promising candidate for next-generation singlephoton sources, due to its chemical and thermal robustness and high brightness at room temperature. The $2 \mathrm{D}$ crystal lattice of $\mathrm{hBN}$ allows for a high extraction efficiency and easy integration into photonic circuits. Here we develop plasma etching techniques with subsequent high-temperature annealing to reliably create defects. We show how different fabrication parameters influence the defect formation probability and the emitter brightness. A full optical characterization reveals the higher quality of the created quantum emitters, represented by a narrow spectrum, short excited state lifetime, and high single-photon purity. We also investigated the photostability on short and very long time scales. We utilize a wet chemically assisted transfer process to reliably transfer the single-photon sources onto arbitrary substrates, demonstrating the feasibility for the integration into scalable photonic quantum information processing networks.
\end{abstract}

KEYWORDS: 2D materials, single photons, fluorescent defect, plasma etching, universal transfer

Sintar ince the rediscovery of graphene, ${ }^{1}$ the field of twodimensional (2D) materials ${ }^{2,3}$ has attracted great interest due to its possible applications in electronics, ${ }^{4}$ optoelectronics, and photonics $^{5}$ as well as advanced sensing ${ }^{6}$ and uses in biophysics. $^{7}$ More recently, the insulating $2 \mathrm{D}$ material hexagonal boron nitride (hBN) has drawn the attention of many researchers due to its ability to host high-luminosity room-temperature single-photon sources (SPSs). ${ }^{8}$ In particular, the outstanding chemical and thermal stability of hBN leads to excellent robustness of the single quantum emitters, which have demonstrated long-term stable operation. ${ }^{9}$ In addition, unlike $\mathrm{NV}$ centers in diamond, monolayered $2 \mathrm{D}$ material based singlephoton sources have an almost ideal out-coupling efficiency of unity, as none of the emitters are surrounded by any high refractive index material and are not affected by Fresnel or total internal reflection. ${ }^{10}$

The single-photon generation mechanism is based on trapping sites at point defects in the crystal lattice, which introduce energy states in the electronic band gap. While this is the generally accepted model, the exact nature of the defects remains unresolved and controversial. First-principles calculations using density functional theory and group theory analysis have already given some insight into the energy level structure. $^{11,12}$ However, the diversity of zero phonon lines (ZPLs), which vary from defect to defect, spanning the full visible spectrum ${ }^{13}$ down to the $U^{14}$ shows that deeper analysis and further experimental investigations are necessary.
Single-photon sources are important for quantum optics, quantum communication, ${ }^{15}$ and optical quantum computing. ${ }^{16}$ These fields allow for the realization of unconditionally secure communication and efficient solutions for mathematically hard problems and simulations that are intractable for even the most powerful classical supercomputers. Protocols in these quantum information processing schemes require narrower emission line widths and shorter excited state lifetimes of the trapped excitons than reported so far for single photons in $\mathrm{hBN}$ at room temperature. Protocols of particular interest include quantum key distribution with single photons (such as BB84 ${ }^{15}$ ) or singlephoton interferometry. ${ }^{19}$ Optical quantum computing requires transform-limited single photons with lifetime-bandwidth products on the order of $1 .{ }^{16}$ To date, single photons generated from $2 \mathrm{D}$ materials have lifetime-bandwidth products ranging from $6 \times 10^{3}$ to $2 \times 10^{4}$ above the transform limit at room temperature. ${ }^{8,13,18-20}$ First attempts of engineering the defect formation have been successful, using either ion irradiation, ${ }^{21}$ chemical etching, ${ }^{22}$ or plasma etching.

In this article, we describe methods to enhance the yield of particularly high quality single-photon emitters in mechanically exfoliated hBN. The primary defect creation mechanism is oxygen plasma etching, ${ }^{9}$ while the defect activation relies on high-temperature thermal annealing. ${ }^{8}$ We investigate how plasma parameters and annealing temperatures influence the

Received: January 31, 2018

Published: April 6, 2018 

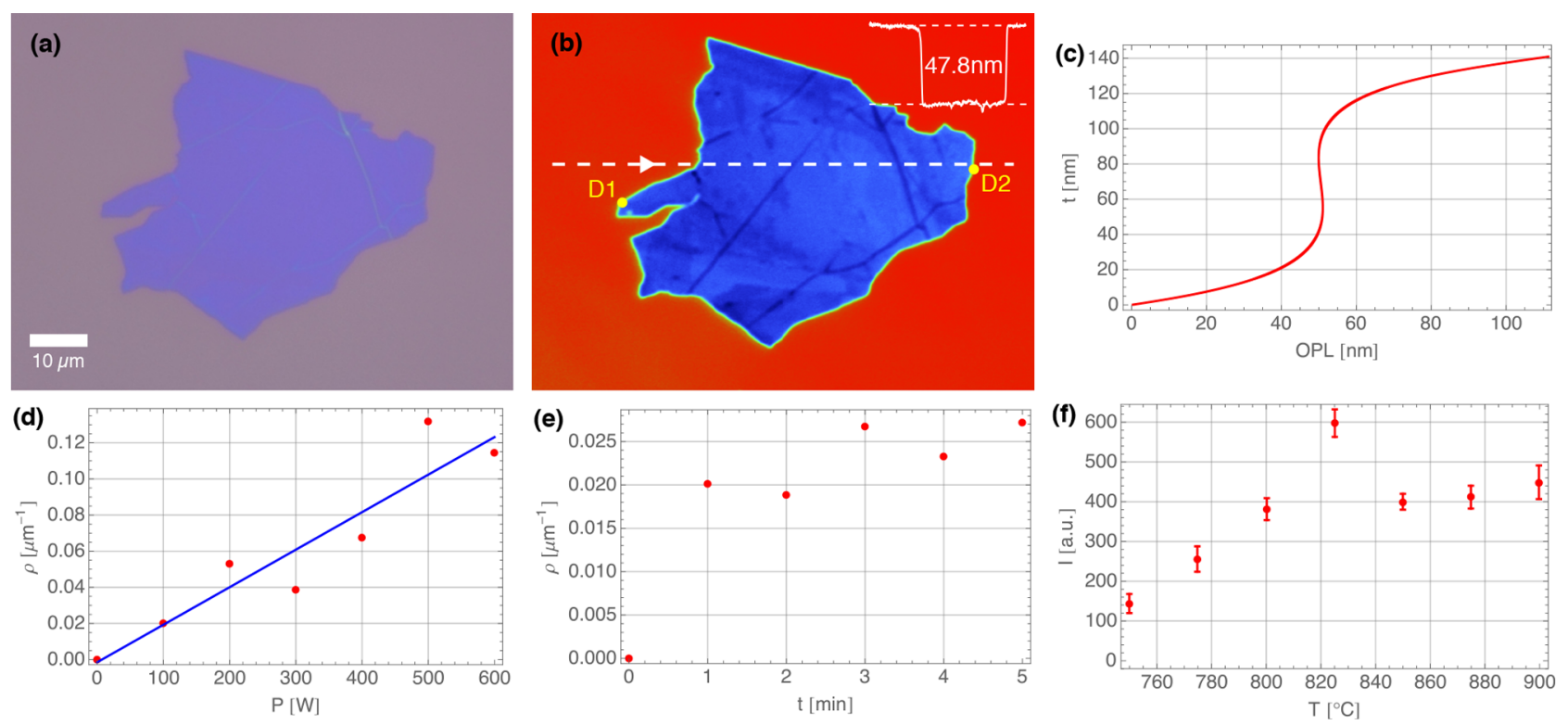

Figure 1. Fabrication parameters. (a) Optical microscope image of an hBN crystal. (b) PSI image of the crystal. The small inset shows the OPL difference along the dashed line. (c) RCWA simulations of the physical thickness as a function of OPL for hBN on $\mathrm{Si} / \mathrm{SiO}_{2}$, calibrated with AFM and PSI measurements. For OPLs around $50 \mathrm{~nm}$ the simulations become ambiguous. (d) The linear defect density increases linearly with the plasma power. The plasma time was $1 \mathrm{~min}$. (e) At a constant plasma power of $100 \mathrm{~W}$ the linear defect density remains approximately constant for different plasma times. (f) Influence of the annealing temperature on the average ZPL brightness. The error bars denote the standard deviation.

formation probability and brightness of the quantum emitters and fully characterize their optical properties in terms of spectral distribution, excited state lifetime, power-dependence, and photostability on short and prolonged time scales. Finally, we employ a universally applicable wet chemical transfer method for transferring the single-photon sources onto arbitrary substrates, allowing for an easy integration into photonic circuits and networks.

\section{DEVICE FABRICATION}

Starting with bulk crystal hexagonal boron nitride, multilayer flakes are mechanically exfoliated onto a viscoelastic foil. Using contrast-enhanced microscopy, thin flakes are selected by optical contrast and transferred by dry $\operatorname{contact}^{23}$ to a $\mathrm{Si}$ substrate with a $280 \mathrm{~nm}$ thermally grown insulating capping layer $\left(\mathrm{SiO}_{2}\right)$. The flake thickness is measured by phase-shift interferometry (PSI), where the optical path length (OPL) through the flake is converted to physical thickness via rigorous coupled-wave analysis (RCWA) simulations. ${ }^{24}$ By measuring the physical flake thickness of a few flakes using atomic force microscopy (AFM), the RCWA simulations of the OPL yield the refractive index of hBN of 1.849 (134) for green light, which matches well previous results. ${ }^{25}$ Knowing the exact refractive index allows for extrapolating the RCWA simulations, so that they serve as a conversion measure between OPL and physical thickness. A microscope image of an example flake is shown in Figure 1(a), together with the corresponding PSI map in Figure 1(b). However, for $\mathrm{hBN}$ on $\mathrm{Si} / \mathrm{SiO}_{2}$ the RCWA simulations yield only reliable results for (physical) flake thicknesses of $<40$ $\mathrm{nm}$, as the simulations give an ambiguous outcome for optical path lengths of $>50 \mathrm{~nm}$, as shown in Figure 1(c). Thus, larger flake thicknesses are measured using AFM. We studied a large variety of flake thicknesses and found crystals with step heights ranging from $4 \mathrm{~nm}$ to roughly $100 \mathrm{~nm}$ were capable of hosting a single-photon emitter.
In order to create the defects, the flakes are treated with an oxygen plasma and thermally annealed to activate the color centers under an argon atmosphere. In the interest of maximizing the quantum emitter yield per flake as well as optimizing the single-photon spectral properties, we varied the plasma power, plasma time, and annealing temperature. After plasma etching and thermal annealing the hBN samples are optically characterized in a confocal microphotoluminescence $(\mu \mathrm{PL})$ system scanning each flake and mapping the PL response. With the laser excitation wavelength being at 522 $\mathrm{nm}(E=2.38 \mathrm{eV})$, the photon energy is well below the band gap of hexagonal boron nitride $\left(E_{\mathrm{g}}=5.955 \mathrm{eV}^{26}\right)$, preventing any delocalized free excitonic emission. With the photon energy of the laser being more than a factor of 2 below the band gap and keeping the excitation power well below saturation (see the next section), multiphoton excitation does not play a major role. As pure hexagonal boron nitride is optically inactive in the visible spectrum, regions with a large PL response are considered as candidates for hosting single-photon emitters. During this confocal mapping, a spectrum has been taken for each scanning position. All measurements have been carried out at room temperature.

For the sake of a fair comparison we define the average linear density of emitters per edge length $\rho=N / L$, as larger flakes are more likely to host defects, independent of the initial plasma parameters. We did not choose to take the areal density, as the emitters are almost exclusively created at the boundaries of the flakes. This is a result of a low defect formation energy at the edges of the flake. We studied plasma powers varying from 100 to $600 \mathrm{~W}$ generated from a microwave field with total plasma times ranging from 1 to $5 \mathrm{~min}$. At this stage in the process, all samples have been subsequently annealed at $850{ }^{\circ} \mathrm{C}$. Figure 1 (d) shows the linear density per unit edge length as a function of plasma power, which exhibits a linear increase in defect density with plasma power (blue fit). This can be explained by the fact that at higher powers the plasma is denser, leading to 

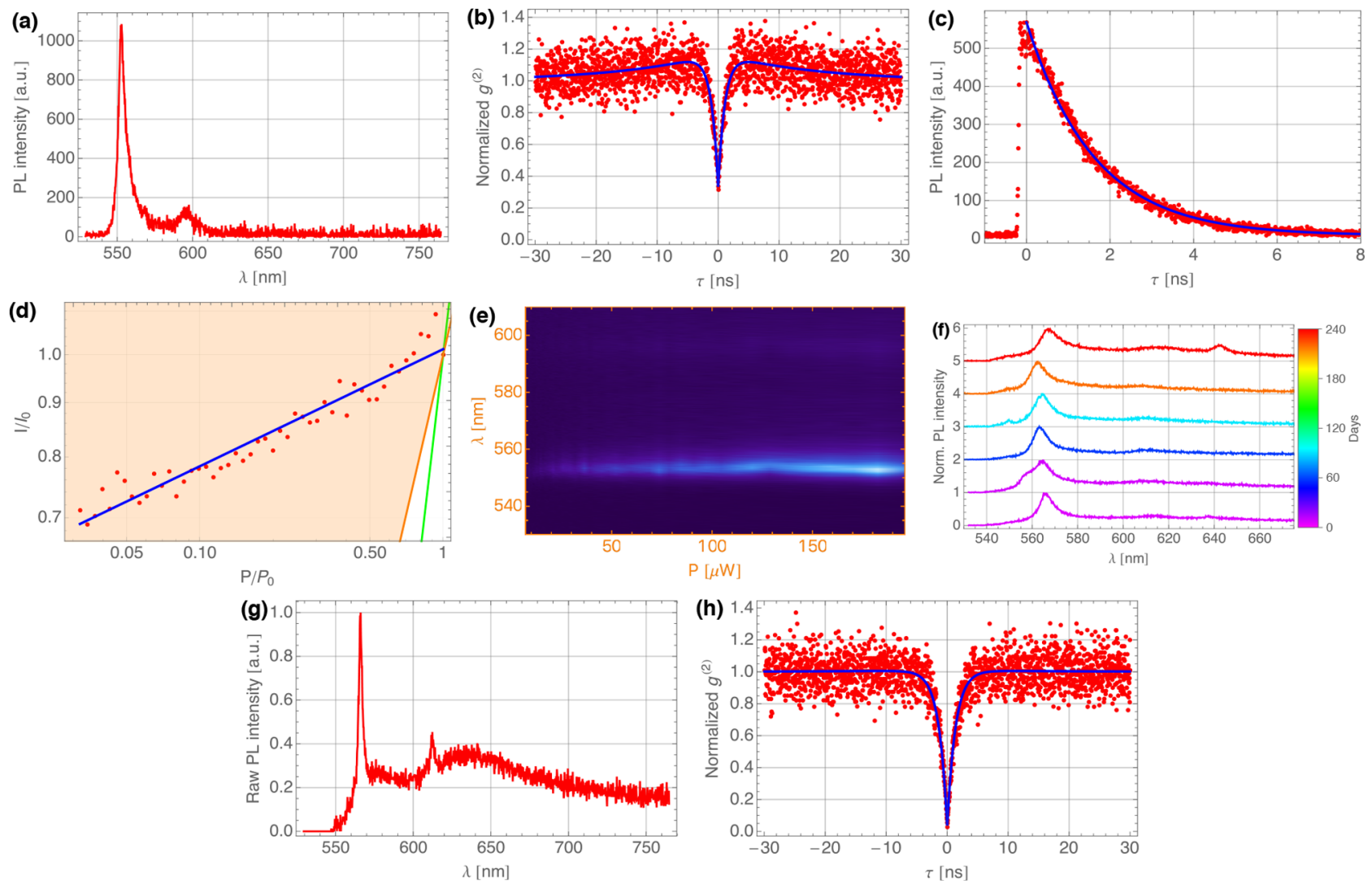

Figure 2. Optical characterization of fabricated defects. (a) Spectrum measured in-reflection after an ultrasteep long-pass filter (opening at $530 \mathrm{~nm}$ ) coupled into a high-resolution spectrometer. Excited at a wavelength of $522 \mathrm{~nm}$, the ZPL is at 553.23(5) nm with a line width of 2.82(10) nm. (b) Second-order correlation function dipping at zero time delay to 0.330(28) (obtained from fit). (c) Time-resolved photoluminescence using an ultrashort pulsed laser, revealing an excited state lifetime of $\tau=1.123(7)$ ns. (d) Log-log plot of the photoluminescence response as a function of excitation power. The orange-shaded area (slope $\alpha<1)$ indicates emission from defects, while the orange line $(\alpha=1)$ corresponds to free excitonic emission and the green line $(\alpha=2)$ biexcitonic emission. The slope $\alpha=0.350(54)<1$ of the linear fit confirms defect emission. (e) Spectrally resolved power-dependence measurement. The emitter showed some power-dependent photobleaching. (f) Long-term stability of a defect over a duration of 8 months (normalized and vertically offset for clarity). The center of the ZPL remains stable within $\pm 2.5 \mathrm{~nm}$, while its line width increases with time. (g) Spectrum of the best single-photon emitter we found with a ZPL at 566.04(4) nm and a line width of 1.31(7) nm. 8.7\% of the emission is into the ZPL. (h) The second-order correlation of the defect with the spectrum shown in (g) dips to 0.033(47) at zero time delay.

the formation of more defects. When keeping the plasma power constant at $100 \mathrm{~W}$ and varying the plasma times as shown in Figure 1(e), the linear defect density remains approximately constant. This is due to etching effects in the oxygen plasma, which is not only creating the defects but also etching the hBN flakes layer by layer. Even though the etching rate is powerdependent, a single layer is etched faster than the time scales investigated here, so longer plasma times tend to remove already formed defects. It is worth noting, however, that the plasma field is highly anisotropic (conditioned by the gas pump, plasma generator, and chamber geometry) and the plasma power varies across the plasma chamber, with the field weakening toward the center of the chamber. Thus, we tried to position the substrates always at the same distance from the chamber walls, but repeating this experiment in a different plasma chamber will require using different plasma powers than the ones reported here.

For the next part of this study we kept the plasma power constant at $100 \mathrm{~W}$ for $1 \mathrm{~min}$ and varied the subsequent annealing temperature from 750 to $900{ }^{\circ} \mathrm{C}$ under an inert argon atmosphere. Annealing under vacuum reduces the defect yield drastically. As the defects are created during the interaction with the plasma and are only optically activated and stabilized during the annealing, we use the average brightness of the ZPL as a figure of merit for a good annealing temperature, while the excitation laser power was kept constant (see Figure 1(f)). The practically usable interval of annealing temperatures spans from 800 to $850{ }^{\circ} \mathrm{C}$, similar to previously reported annealing temperatures. ${ }^{8,13,27}$ Lower annealing temperatures lead to weak ZPLs, where the defects are not fully optically activated, while higher annealing temperatures cause the defects to diffuse too much. Especially the latter effect is present for long annealing times as well; hence we employed rapid thermal annealing (RTA) instead of standard furnace annealing. We note that, unlike in previous reports, ${ }^{8,13}$ annealing in a tube furnace did not yield any bright and stable single-photon emitters in our experiments.

\section{OPTICAL CHARACTERIZATION}

We now turn to a full characterization of the single-photon emitters. The sample crystal shown in Figure 1(a) hosts two defects, with their positions labeled D1 and D2 in Figure 1(b). The spectrum of D1 is shown in Figure 2(a), and from a fit we extract the ZPL at a wavelength of 553.23(5) $\mathrm{nm}$ and a line width of $2.82(10) \mathrm{nm}$. Unless stated otherwise, we use a $95 \%$ confidence interval for the uncertainties, calculated using Monte Carlo simulation methods. This defect emitted $82.4 \%$ into its ZPL. Using a Hanbury Brown and Twiss (HBT)-type 
interferometer, we measure the second-order correlation function (see Figure 2(b)), with $g^{(2)}(\tau=0)$ dipping to $0.330(28)$, obtained by fitting a three-level system with ground and excited states as well as a metastable shelving state:

$$
g^{(2)}(\tau)=1-A \mathrm{e}^{-|\tau-\mu| / t_{1}}+B \mathrm{e}^{-|\tau-\mu| / t_{2}}
$$

where $t_{1}$ and $t_{2}$ are the excited and metastable state lifetimes, respectively, $\mu$ accounts for different electrical and optical path lengths in the HBT interferometer, and $A$ and $B$ are the antibunching and bunching amplitudes. The experimental data have been normalized such that for very long time delays $g^{(2)}(\tau$ $\rightarrow \infty)=1$. In all correlation function measurements no background correction has been applied, as these measurements are not yet dark count limited; the dark counts from the used detectors are very low compared to the single-photon count rate (see Methods). The $\mu \mathrm{PL}$ system is equipped with an ultrashort pulsed laser with a $300 \mathrm{fs}$ pulse length at a repetition rate of $20.8 \mathrm{MHz}$, allowing us to measure the exciton lifetime as well, with the lifetime of D1 shown in Figure 2(c). A fit of a single-exponential decay reveals an excited state lifetime $\tau$ of 1.123(7) ns. The shelving state lifetime is not accessible from this measurement due to its weak transition and longer lifetime. However, the decay time is consistent with the correlation function measurements: From the fit of the $g^{(2)}$ function we obtain $t_{1}=1.100(134) \mathrm{ns}$ and $t_{2}=15.441(4168) \mathrm{ns}$ (all other fit parameters are summarized in the Supporting Information S1). Together with its line width $\Delta \nu=\frac{c \Delta \lambda}{\lambda^{2}}$ this yields a lifetime-bandwidth product of 3102 , still far above the transform limit, but better than any reported emitter in hBN so far. In addition we measured the PL intensity as a function of excitation power, which is described by

$$
I(P)=\frac{I_{\mathrm{sat}} P}{P+P_{\mathrm{sat}}}+I_{\mathrm{d}}
$$

with $I_{\text {sat }}$ and $P_{\text {sat }}$ being the saturation intensity and power, respectively, and $I_{\mathrm{d}}$ is the dark count intensity. From a fit we extract $P_{\text {sat }}=142.6(685) \mu \mathrm{W}$. Together with a focal spot diameter of $0.67 \mu \mathrm{m}$ and a duty cycle of $6.24 \times 10^{-6}$ this amounts to a peak intensity of $1.62 \mathrm{GW} \mathrm{cm}^{-2}$, which is still below the damage threshold. Figure 2(d) shows this measurement (red dots) together with the fit (blue line) on a log-log scale, which confirms the defect nature of the emission: A slope of $\alpha=1$ indicates free excitonic emission (orange line), while $\alpha$ $=2$ reveals the presence of biexcitons (green line) and $\alpha<1$ verifies trapped excitons from a defect (orange-shaded area). ${ }^{28}$ The power-dependence of D1 has a slope of $\alpha=0.350$ (54), clearly in the defect emitter region. Furthermore, we measured the power-dependent photostability, which is shown in Figure 2(e). Defect D1 showed some power- as well as timedependent photobleaching. The power-dependent photobleaching causes the deviations from the linear fit in Figure 2 (d) and is also the reason for the large confidence interval on $P_{\text {sat }}$. However, we have also found emitters that were photostable. Finally, we also look at the long-term stability, meaning repeating all measurements above for a subset of samples over a time span of more than 8 months. In between measurements the samples were stored under normal atmosphere in air. Figure 2(f) shows the spectra for different days, all normalized and offset vertically for clarity. With the center of the ZPL being constant within $\pm 2.5 \mathrm{~nm}$, its line width increases over time from $4.38(13) \mathrm{nm}$ to $6.61(25) \mathrm{nm}$. Other optical properties such as $\tau, \alpha$, and $g^{(2)}(0)$ are varying as well, without showing a clear trend in the case of $\alpha$ and $g^{(2)}(0)$ (see Supporting Information S2), while $\tau$ shortens with an increase in line width (see also the next section). The variations can be explained by the fact that $2 \mathrm{D}$ materials typically oxidize in an ambient environment. The stability in air is ultimately controlled by the oxygen dissociative absorption barrier and is also affected by defects present. As the host crystal is very thin and the interactions within the crystal are strong, already small variations can cause large changes in the photophysical properties. Isolating the crystal from any coupling to the environment, such as through encapsulation, can improve the long-term stability, but the influence of the encapsulation layer must be investigated. Nevertheless, the defects maintain their single-photon emission properties on short and very long time scales and at the same time keep the photophysical properties (for a $2 \mathrm{D}$ material) constant within the reported limits.

\section{CORRELATING OPTICAL PROPERTIES}

The optical properties as described in the previous section are by no means representative for all defects, but are typical photophysical properties. The optical properties in terms of spectral distribution, excited state lifetime, power-dependence, photostability, and second-order correlation function vary not only from flake to flake but also from defect to defect hosted by the same flake. As reported previously, ${ }^{13}$ the ZPLs cover the full visible spectrum below the excitation photon energy. In our experiments the quantum emitter ZPLs span a range from 550 to $720 \mathrm{~nm}$, with the lower limit set by a long-pass filter used to filter out the excitation laser and the upper limit set by the spectrometer bandwidth. The line widths vary from as low as $1.31(7) \mathrm{nm}$ (see Figure 2(g)) to 11.6(4) $\mathrm{nm}$ at room temperature, while the exciton lifetimes span a smaller range from $294(3)$ ps to $1.32(1) \mathrm{ns}$ [1.31 nm line width and $1.32 \mathrm{~ns}$ lifetime are not from the same defect; the same holds for 11.6 $\mathrm{nm}$ and $294 \mathrm{ps}$ ]. This is more than 1 order of magnitude faster than any previously reported excited state lifetime in $\mathrm{hBN}$; in fact, all of the defects have shorter lifetimes than the fastest previously reported ones (see also Supporting Information S4). The single-photon purities characterized by $g^{(2)}(0)$ vary from $0.033(47)$ to $0.480(38)$ (excluding any emitter with $g^{(2)}(0)>$ 0.5 , which are considered ensembles). A single-photon purity with $g^{(2)}(0)=0.033(47)$ (see Figure $2(\mathrm{~h})$ ) in $\mathrm{hBN}$ is only matched by emitters coupled to plasmonic nanocavity arrays, ${ }^{29}$ with $g^{(2)}(0)=0.02-0.04$. This defect has a time-bandwidth product of 1389 . The slopes of the power saturation vary from $0.290(56)$ to 0.942 (43) for different defects. Across all defects, the optical properties are randomly distributed with the exception of the zero phonon line, which with a 53\% chance is between 550 and $570 \mathrm{~nm}$ (see Supporting Information S4).

The natural question then arises whether there is any correlation between the optical properties and especially between the optical properties and the fabrication parameters or geometrical features of the host crystal flakes. By studying a large variety of flakes and cross-correlating optical properties, we found that a narrow line width correlates with a longer excited state lifetime, even though the single photons are still above the transform limit (see Supporting Information S3). The smallest time-bandwidth product was 807 at room temperature, which is 1 order of magnitude smaller than any previously reported value. The mean of 3782 for this product shows the higher quality of the emitters, compared to emitters fabricated by other methods. ${ }^{8,13,18-20}$ So far it seems that neither the fabrication parameters nor the physical crystal 

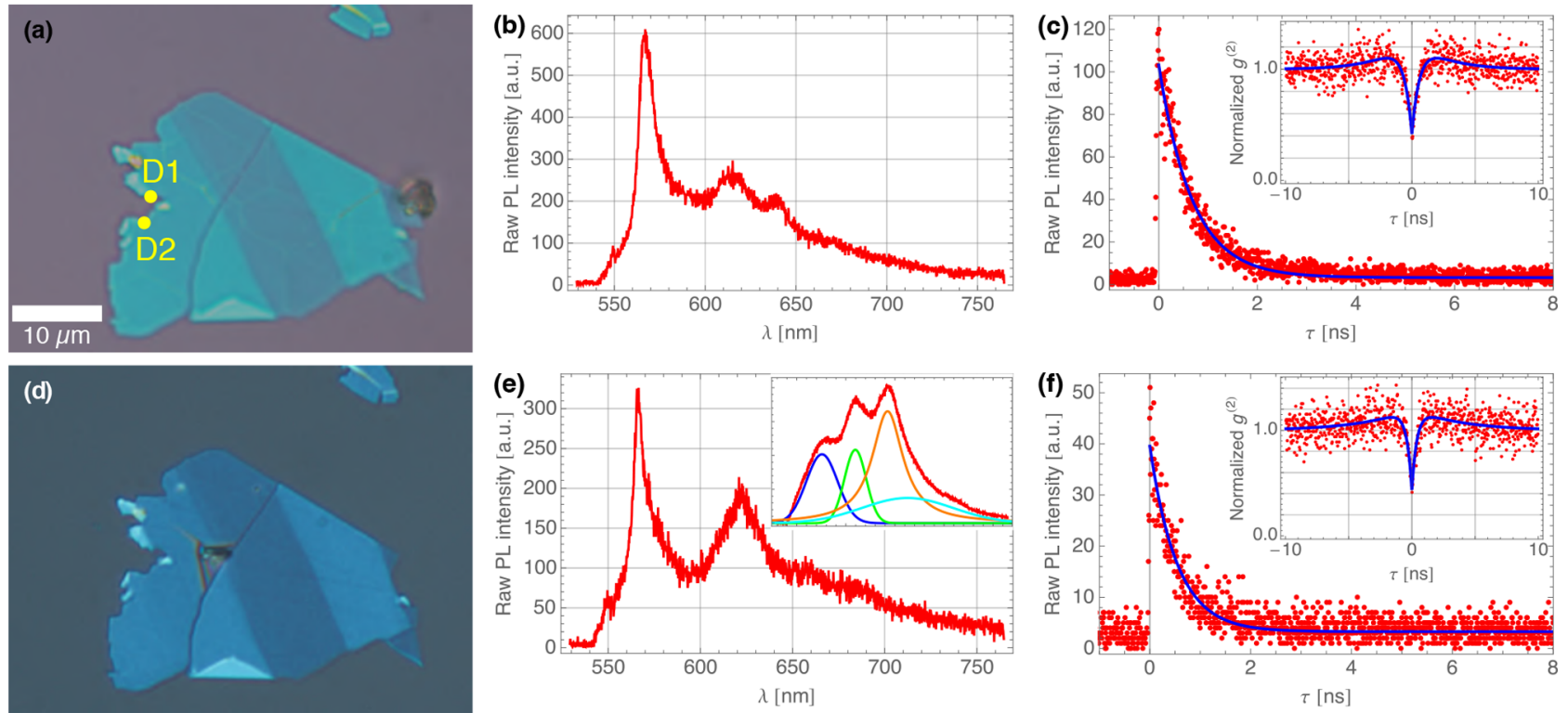

Figure 3. Deterministic transfer of a quantum emitter. (a, d) Optical microscope image before and after the transfer at $1000 \times$ magnification. The locations of defects D1 and D2 are marked with yellow dots. (b, e) Spectrum of D2 before and after the transfer. The ZPL peaks at 567.61(8) nm, which is marginally blue-shifted to 567.39(13) nm after the transfer. The small inset in (e) shows the spectrum before the plasma cleaning: From a fit four peaks can be extracted, which can be assigned to PVP (blue), NVP (green), and PVA (orange). The horizontal axis has the same scale as the large spectrum, while its vertical axis is on a much larger scale. (c, f) Time-resolved photoluminescence response before and after the transfer. The excited state lifetime is $\tau=468(8)$ ps and is shortened to $\tau=375(15)$ ps after the transfer. The purity remains approximately constant (small insets), with $g^{(2)}=0.416(55)$ and $g^{(2)}=0.433(57)$ before and after the transfer, respectively.

thickness at the defect position has any influence on the emission spectra, lifetimes, purities, or $\alpha$. The latter demonstrates that the interaction of the in-plane dipole with surrounding layers is probably small. Even though it remains obscure why defects formed by plasma etching of the host crystals perform better and have particularly short lifetimes, we have seen that this process reliably creates a large number of higher quality single-photon emitters (see also Supporting Information S4). In total we studied more than 300 flakes hosting more than 200 defects. Each flake hosted between 0 and 7 defects, with the average number being 2.55 (not counting the flakes hosting no defect).

\section{DETERMINISTIC TRANSFER OF QUANTUM EMITTERS}

Finally, we demonstrate a deterministic transfer of the quantum emitters onto arbitrary substrates. The $\mathrm{Si} / \mathrm{SiO}_{2}$ substrates, on which the hBN flakes are bonded by van der Waals force, are good for characterization, but from an application point of view, the single-photon emitter must be integrated into photonic devices or networks. It is possible to transfer the flakes directly from the polymer foil onto the photonic device before plasma treatment and thermal annealing, but as the defects are formed at random positions, this is not favorable. Furthermore, the high annealing temperature may damage integrated singlephoton devices. For monolayer transition metal dichalcogenides (TMDs) it has been demonstrated that stress induced by nanopillars allows the formation and precise positioning of quantum emitter arrays. ${ }^{30}$ Here we employ a wet chemical transfer method developed to transfer TMDs from $\mathrm{SiO}_{2}$ onto other substrates. ${ }^{31}$ The technique is based on using a twocomponent polymer mediator, which consists of polyvinylpyrrolidone (PVP)/N-vinylpyrrodoline (NVP) and poly(vinyl alcohol) (PVA), where the PVP/NVP provides good adhesion to the crystal, while the PVA reinforces the mechanical strength of the PVP film. However, as the hBN flakes have considerably more layers compared to monolayered crystals, we adapted the polymer concentrations (see Methods). The solutions are spin coated onto the sample, and the resulting polymer carpet can be pressed onto a new viscoelastic foil, from which it can be transferred to its new substrate. Then the PVP/NVP dissolves easily in water.

After the transfer all hBN crystals exhibited a strong broadband PL emission with peak maxima at 575.5, 609.6, 642.5, and $662.9 \mathrm{~nm}$ (see Figure 3(e), small inset), making it impossible to resolve the single-photon ZPL. This PL was traced back to polymer chains remaining on the hBN, with PVP peaking around $576.4 \mathrm{~nm}, \mathrm{NVP}$ peaking around $605.2 \mathrm{~nm}$, and PVA peaking around $619.6 \mathrm{~nm}$. The hBN red-shifts the PVA peak, explaining the third large background peak. The polymers adhered to the hBN even after soaking in distilled water for 14 $\mathrm{h}$ at elevated temperatures of $60{ }^{\circ} \mathrm{C}$ for accelerated solution, meaning that the adhesion of the polymer to the $\mathrm{hBN}$ is stronger than its solubility in water. The solubility in other polar protic solvents (mostly alcohols) turned out to be too low as well. Finally, using low power oxygen plasma cleaning, the polymers can be fully removed. However, great care must be taken such that the $\mathrm{hBN}$ itself is not etched. It shall be mentioned that the $2 \mathrm{D}$ materials community developed a great toolbox of other transfer techniques, for example utilizing different polymers for the pick-up. ${ }^{32}$ Using different polymers for the transfer might not introduce fluorescent residues.

We characterized the single-photon emission properties before and after a full transfer cycle. The example flake presented here hosted two defects, which both have survived the transfer. An optical microscope image prior to the transfer is shown in Figure 3(a), with both defects marked with yellow dots labeled D1 and D2. The spectrum and lifetime before the 


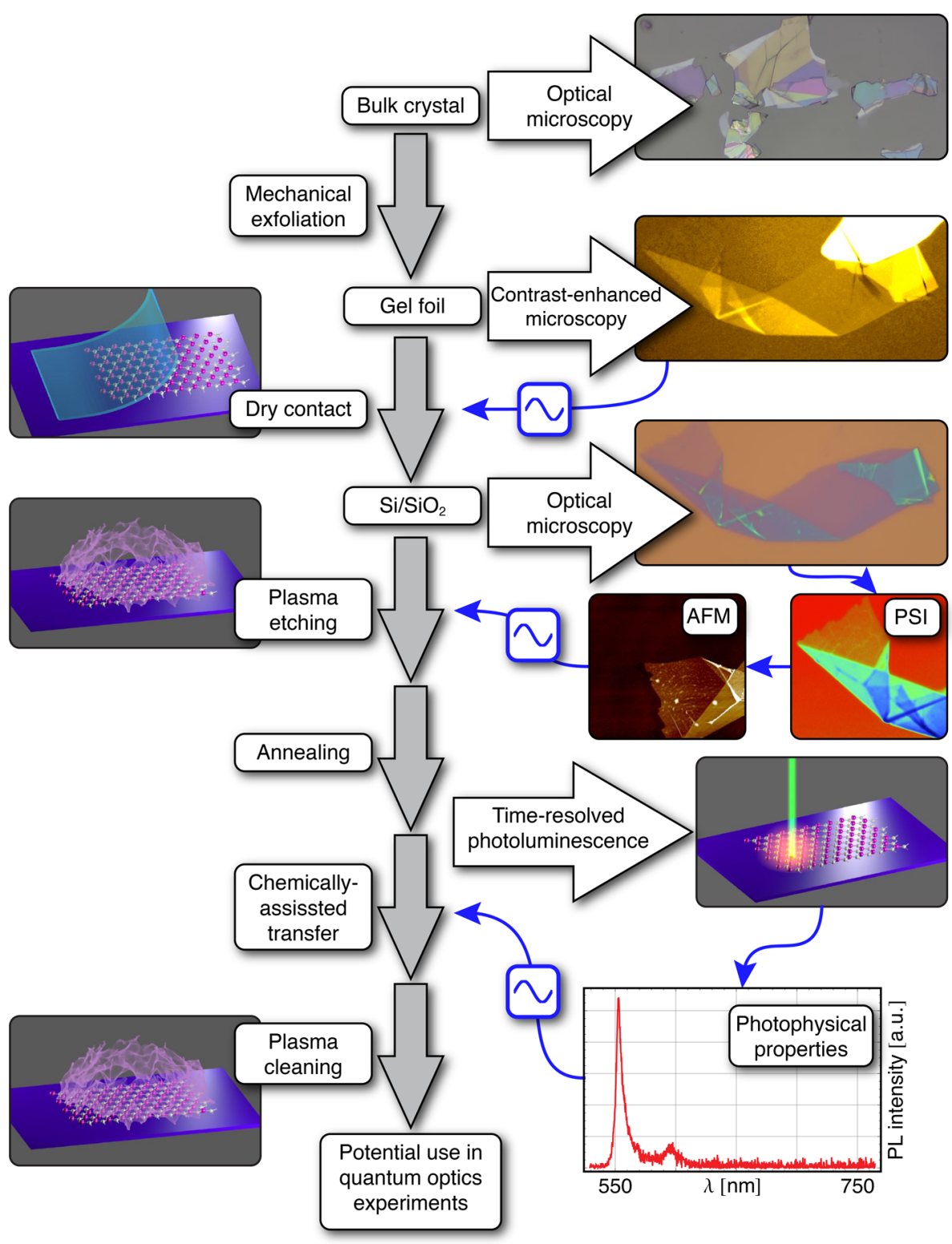

Figure 4. Full process cycle for hBN quantum emitter fabrication. The left column shows the processes introduced, and the right column shows the characterization and selection of samples. The central column shows the development of the sample. $\mathrm{hBN}$ flakes are initially exfoliated from the bulk crystal. The flakes are first optically identified using contrast-enhanced microscopy. Appropriate flakes are selected for a dry contact transfer to Si/ $\mathrm{SiO}_{2}$ substrates. The transferred flakes are again selected for flake thickness measurement using phase-shift interferometry (PSI). Depending on the optical path length value, the exact physical thickness is measured using atomic force microscopy (AFM). Crystals with thicknesses in the suitable range undergo oxygen plasma etching and thermal annealing, after which they are fully optically characterized in a time-resolved photoluminescence (TRPL) setup. Flakes with good photophysical properties could be transferred onto waveguides or fibers, where the single-photon sources could be used in a potential quantum optics experiment.

transfer process are shown in Figure 3(b) and (c), respectively. The small inset in (c) is the second-order correlation function. Prior to contact with the polymers the ZPL was at 567.61(8) $\mathrm{nm}$ with a line width of $4.99(17) \mathrm{nm}$ and the lifetime was $468(8)$ ps with $g^{(2)}(0)=0.416(55)$. After the full transfer process including plasma cleaning, the microscope image shows additional cracks in the host crystal (see Figure 3(d)), but the part with the single photon emitter is not affected. Intermediate microscope images after each step show that the cracks are not caused by the polymers, but rather occur during peeling off the polymer carpet from the initial substrate. Repeated experiments proved that this happens only where the host crystal already has cracks prior to the transfer process (see Figure 3(a)). Defects that are close to such cracks are therefore not suitable for this transfer method. The ZPL is slightly blue-shifted to 567.39(13) $\mathrm{nm}$ with the line width unchanged, as shown in Figure 3(e). The defect's ZPL peak brightness is only $53.47 \%$ of the brightness before the transfer, with the phonon sideband approximately equally strong compared to prior to the transfer. This results in $13.0 \%$ of the light being emitted into the ZPL, which was $24.9 \%$ prior to the transfer. Narrow filtering of the ZPL nevertheless allowed measurement of the excited state lifetime, which is shortened to $375(15)$ ps with $g^{(2)}(0)$ increased to $0.433(57)$. The shortening of the lifetime might be due to small alterations of the host crystal structure (meaning the defect's environment) during the plasma 
cleaning. The same might apply for the reduction of emission into the ZPL. Further optimization of the transfer process might increase the overall performance of the transfer cycle, especially in reference to the loss in brightness of the ZPL. However, so far every transfer cycle was successful. A full process cycle starting with the bulk $\mathrm{hBN}$ to the chemical transfer process is shown in Figure 4.

\section{CONCLUSION}

The fabrication techniques reported here demonstrate how oxygen plasma etching can create color centers in exfoliated multilayer hexagonal boron nitride that form, after optical activation through thermal annealing, stable single-photon emitters. The emitters show excellent optical properties in terms of narrow line widths and lifetimes as short as 294 ps, which are 1 order of magnitude shorter than reported so far, allowing for a high operational bandwidth of the single-photon source. Extended statistics show that many emitters with these photophysical properties are created, almost all of which have a lower time-bandwidth product at room temperature than previously reported. The emitters are also very robust, maintaining their single-photon emission capabilities over the time frame of this experimental work, which is currently 8 months. However, due to the substantial variation of even basic optical properties such as ZPL position in the spectrum or excitonic lifetime even from emitter to emitter hosted by the same flake, the exact nature of the defect remains obscure. This indicates that different defects are present, which is additionally emphasized by the fact that we did not find a correlation between single-photon emission properties and the fabrication parameters or geometrical features of the host crystals. Finally, we have also demonstrated that these quantum emitters can be transferred reliably, while preserving their single-photon emission capabilities. This technique allows the integration of the single-photon sources into photonic circuits and networks, such as fibers and waveguide platforms. Thus, this provides a building block for next-generation quantum information processing. Only commonly available nanofrabrication processes have been used, making the fabrication easy and repeatable.

Note added: While under review we became aware of a recent related work. ${ }^{33}$

\section{METHODS}

Sample Fabrication. The bulk crystal was acquired from HQGraphene, exfoliated to Gel-Pak WF-40-X4, and transferred by dry contact to $\mathrm{Si} / \mathrm{SiO}_{2}$ substrates ( $280 \mathrm{~nm}$ thermally grown). After thickness measurements using PSI or AFM the samples were treated with a microwave plasmas of different powers and lengths. The pressure for all plasmas was set to $0.3 \mathrm{mbar}$ at an oxygen gas flow rate of $300 \mathrm{~cm}^{3} / \mathrm{min}$ at room temperature. Subsequent annealing at different temperatures for a few minutes under an argon atmosphere took place in a rapid thermal annealer. After multiple evacuations of any residual gases the Ar flow was set to $500 \mathrm{~cm}^{3} / \mathrm{min}$. After the annealing the samples cooled at their natural cooling rate, without keeping the cooling rate at a maximal value.

Optical Characterization. The home-built microphotoluminescence setup used an ultrashort pulsed frequency-doubled $1044 \mathrm{~nm}$ laser (High Q Laser URDM) focused down to the diffraction limit by an Olympus $100 \times / \mathrm{NA}=0.9$ dry objective. The samples were mounted on Newport piezoscanning stages with $0.2 \mu \mathrm{m}$ resolution. The emission, collected through the same objective, was frequency-filtered (Semrock RazorEdge ultrasteep long-pass edge filter) to dump the excitation light and guided to a spectrometer (Princeton Instruments SpectraPro). The pulse length of the laser was $300 \mathrm{fs}$ at a repetition rate of $20.8 \mathrm{MHz}$. The laser pulses were split into trigger and excitation beams, and a single-photon counter (Micro Photon Devices) detected the emitted photons after the trigger signal. The temporal correlation between trigger time and single-photon arrival time was given by a PicoHarp 300 . The second-order correlation function was measured in a different setup using a $512 \mathrm{~nm}$ diode laser. This setup was equipped with a nanopositioning stage and a spectrometer as well. The single-photon counters used in this setup are 2 PerkinElmer SPCM-AQR-16, which are ultralow dark count single-photon counting modules with dark count rates as low as $20 \mathrm{~s}^{-1}$.

Transfer Process. The method was developed in ref 31, but the polymer concentrations for hBN have been adjusted. The target substrates were initially plasma cleaned. The samples were prebaked at $80{ }^{\circ} \mathrm{C}$ for $1-2 \mathrm{~min}$ and subsequently spin coated at $2000 \mathrm{rpm}$ for $50 \mathrm{~s}$ with a PVP/NVP solution (1.7 g of $\mathrm{PVP}+1.5 \mathrm{~mL}$ of NVP $+0.75 \mathrm{~mL}$ of $\mathrm{H}_{2} \mathrm{O}+7 \mathrm{~mL}$ of ethanol, dissolved at $40{ }^{\circ} \mathrm{C}$ and filtered) and then postbaked for $1-2$ min. This was repeated with a 9\% PVA solution (molecular weight in DI water). The resulting polymer was peeled off at the edges using a scalpel and then pressed onto a new gel foil (Gel-Pak WF-40-X4), and the polymer carpet remained on the foil. Next, the crystal was transferred to a new sample by standard means. After baking the new substrate with the gel foil attached at $120{ }^{\circ} \mathrm{C}$ for $3-5 \mathrm{~min}$, the polymer remained on the new substrate and was dissolved in DI water for $1 \mathrm{~h}$ and rinsed with IPA. A final plasma cleaning step removed remaining polymer chains.

\section{ASSOCIATED CONTENT}

\section{Supporting Information}

The Supporting Information is available free of charge on the ACS Publications website at DOI: 10.1021/acsphotonics.8b00127.

(1) Correlation function fit parameters; (2) detailed photophysical properties of a defect over the time frame of this study; (3) more detailed analysis of the correlation between line width and lifetime; (4) extended statistics showing the distribution of photophysical properties for a series of quantum emitters (PDF)

\section{AUTHOR INFORMATION}

\section{Corresponding Authors}

*E-mail: Tobias.Vogl@anu.edu.au.

*E-mail: Ping.Lam@anu.edu.au.

ORCID *

Tobias Vogl: 0000-0002-0993-0648

Yuerui Lu: 0000-0001-6131-3906

\section{Notes}

The authors declare no competing financial interest.

\section{ACKNOWLEDGMENTS}

This work was funded by the Australian Research Council (CE110001027, FL150100019, and DE140100805). We thank the ACT Node of the Australian National Fabrication Facility 
for access to their nano- and microfabrication facilities, particularly Kaushal Vora for technical support with the RTA and Fouad Karouta for technical support with the plasma system. We also thank Hark Hoe Tan for access to the TRPL system.

\section{REFERENCES}

(1) Novoselov, K. S.; Geim, A. K.; Morozov, S. V.; Jiang, D.; Zhang, Y.; Dubonos, S. V.; Grigorieva, I. V.; Firsov, A. A. Electric Field Effect in Atomically Thin Carbon Films. Science 2004, 306, 666-669.

(2) Lin, Z.; et al. 2D materials advances: from large scale synthesis and controlled heterostructures to improved characterization techniques, defects and applications. 2D Mater. 2016, 3, 042001.

(3) Manzeli, S.; Ovchinnikov, D.; Pasquier, D.; Yazyev, O. V.; Kis, A. 2D transition metal dichalcogenides. Nat. Rev. Mats. 2017, 2, 1703310.1038/natrevmats.2017.33.

(4) Wachter, S.; Polyushkin, D. K.; Bethge, O.; Mueller, T. A microprocessor based on a two-dimensional semiconductor. Nat. Commun. 2017, 8, 1494810.1038/ncomms14948.

(5) Ponraj, J. S.; Xu, Z.-Q.; Dhanabalan, S. C.; Mu, H.; Wang, Y.; Yuan, J.; Li, P.; Thakur, S.; Ashrafi, M.; Mccoubrey, K.; Zhang, Y.; Li, S.; Zhang, H.; Bao, Q. Photonics and optoelectronics of twodimensional materials beyond graphene. Nanotechnology 2016, 27, 462001.

(6) Yang, S.; Jiang, C.; Wei, S.-H. Gas sensing in 2D materials. Appl. Phys. Rev. 2017, 4, 021304.

(7) Thomas, S.; Rajan, A. C.; Rezapour, M. R.; Kim, K. S. In Search of a Two-Dimensional Material for DNA Sequencing. J. Phys. Chem. C 2014, 118, 10855-10858.

(8) Tran, T. T.; Bray, K.; Ford, M. J.; Toth, M.; Aharonovich, I. Quantum emission from hexagonal boron nitride monolayers. Nat. Nanotechnol. 2016, 11, 37-41.

(9) Vogl, T.; Lu, Y.; Lam, P. K. Room temperature single photon source using fiber-integrated hexagonal boron nitride. J. Phys. D: Appl. Phys. 2017, 50, 295101.

(10) Gould, M.; Schmidgall, E. R.; Dadgostar, S.; Hatami, F.; Fu, K.M. C. Efficient Extraction of Zero-Phonon-Line Photons from Single Nitrogen-Vacancy Centers in an Integrated GaP-on-Diamond Platform. Phys. Rev. Appl. 2016, 6, 011001.

(11) Tawfik, S. A.; Ali, S.; Fronzi, M.; Kianinia, M.; Tran, T. T.; Stampfl, C.; Aharonovich, I.; Toth, M.; Ford, M. J. First-principles investigation of quantum emission from hBN defects. Nanoscale 2017, 9, 13575-13582.

(12) Abdi, M.; Chou, J.-P.; Gali, A.; Plenio, M. B. Color centers in hexagonal boron nitride monolayers: A group theory and ab initio analysis. arXiv:1709.05414, 2017.

(13) Tran, T. T.; Elbadawi, C.; Totonjian, D.; Lobo, C. J.; Grosso, G.; Moon, H.; Englund, D. R.; Ford, M. J.; Aharonovich, I.; Toth, M. Robust Multicolor Single Photon Emission from Point Defects in Hexagonal Boron Nitride. ACS Nano 2016, 10, 7331-7338.

(14) Bourrellier, R.; Meuret, S.; Tararan, A.; Stéphan, O.; Kociak, M.; Tizei, L. H. G.; Zobelli, A. Bright UV Single Photon Emission at Point Defects in h-BN. Nano Lett. 2016, 16, 4317-4321.

(15) Gisin, N.; Ribordy, G.; Tittel, W.; Zbinden, H. Quantum cryptography. Rev. Mod. Phys. 2002, 74, 145-195.

(16) Kok, P.; Munro, W. J.; Nemoto, K.; Ralph, T. C.; Dowling, J. P.; Milburn, G. J. Linear optical quantum computing with photonic qubits. Rev. Mod. Phys. 2007, 79, 135-174.

(17) Elitzur, A. C.; Vaidman, L. Quantum mechanical interaction-free measurements. Found. Phys. 1993, 23, 987.

(18) Schell, A. W.; Takashima, H.; Tran, T. T.; Aharonovich, I.; Takeuchi, S. Coupling Quantum Emitters in 2D Materials with Tapered Fibers. ACS Photonics 2017, 4, 761-767.

(19) Tran, T. T.; Kianinia, M.; Nguyen, M.; Kim, S.; Xu, Z.-Q.; Kubanek, A.; Toth, M.; Aharonovich, I. Resonant Excitation of Quantum Emitters in Hexagonal Boron Nitride. ACS Photonics 2018, 5, 295-300.
(20) Sontheimer, B.; Braun, M.; Nikolay, N.; Sadzak, N.; Aharonovich, I.; Benson, O. Photodynamics of quantum emitters in hexagonal boron nitride revealed by low-temperature spectroscopy. Phys. Rev. B: Condens. Matter Mater. Phys. 2017, 96, 121202.

(21) Choi, S.; Tran, T. T.; Elbadawi, C.; Lobo, C.; Wang, X.; Juodkazis, S.; Seniutinas, G.; Toth, M.; Aharonovich, I. Engineering and Localization of Quantum Emitters in Large Hexagonal Boron Nitride Layers. ACS Appl. Mater. Interfaces 2016, 8, 29642-29648.

(22) Chejanovsky, N.; Rezai, M.; Paolucci, F.; Kim, Y.; Rendler, T.; Rouabeh, W.; Fávaro de Oliveira, F.; Herlinger, P.; Denisenko, A.; Yang, S.; Gerhardt, I.; Finkler, A.; Smet, J. H.; Wrachtrup, J. Structural Attributes and Photodynamics of Visible Spectrum Quantum Emitters in Hexagonal Boron Nitride. Nano Lett. 2016, 16, 7037-7045.

(23) Castellanos-Gomez, A.; Buscema, M.; Molenaar, R.; Singh, V.; Janssen, L.; van der Zant, H. S. J.; Steele, G. A. Deterministic transfer of two-dimensional materials by all-dry viscoelastic stamping. $2 D$ Mater. 2014, 1, 011002.

(24) Yang, J.; Wang, Z.; Wang, F.; Xu, R.; Tao, J.; Zhang, S.; Qin, Q.; Luther-Davies, B.; Jagadisch, C.; Lu, Y. Atomically thin optical lenses and gratings. Light: Sci. Appl. 2002, 5, e16046.

(25) Schubert, M.; Rheinländer, B.; Franke, E.; Neumann, H.; Hahn, J.; Röder, M.; Richter, F. Anisotropy of boron nitride thin-film reflectivity spectra by generalized ellipsometry. Appl. Phys. Lett. 1997, $70,1819-1821$

(26) Cassabois, G.; Valvin, P.; Gil, B. Hexagonal boron nitride is an indirect bandgap semiconductor. Nat. Photonics 2016, 10, 262-266.

(27) Dietrich, A.; Bürk, M.; Steiger, E. S.; Antoniuk, L.; Tran, T. T.; Nguyen, M.; Aharanovich, I.; Jelezko, F.; Kubanek, A. Narrowband quantum emitters over large spectral range with Fourier-limited linewidth in hexagonal boron nitride. arXiv:1712.06938, 2017.

(28) Miyauchi, Y.; Iwamura, M.; Mouri, S.; Kawazoe, T.; Ohtsu, M.; Matsuda, K. Brightening of excitons in carbon nanotubes on dimensionality modification. Nat. Photonics 2013, 7, 715-719.

(29) Tran, T. T.; Wang, D.; Xu, Z.-Q.; Yang, A.; Toth, M.; Odom, T. W.; Aharonovich, I. Deterministic Coupling of Quantum Emitters in 2D Materials to Plasmonic Nanocavity Arrays. Nano Lett. 2017, 17, 2634-2639.

(30) Palacios-Berraquero, C.; Kara, D. M.; Montblanch, A. R.-P.; Barbone, M.; Latawiec, P.; Yoon, D.; Ott, A. K.; Loncar, M.; Ferrari, A. C.; Atatüre, M. Large-scale quantum emitter arrays in atomically thin emiconductors. Nat. Commun. 2017, 8, 1509310.1038/ncomms15093.

(31) Lu, Z.; Sun, L.; Xu, G.; Zheng, J.; Zhang, Q.; Wang, J.; Jiao, L. Universal Transfer and Stacking of Chemical Vapor Deposition Grown Two-Dimensional Atomic Layers with Water-Soluble Polymer Mediator. ACS Nano 2016, 10, 5237-5242.

(32) Pizzocchero, F.; Gammelgaard, L.; Jessen, B. S.; Caridad, J. M.; Wang, L.; Hone, J.; Boggild, P.; Booth, T. J. The hot pick-up technique for batch assembly of van der Waals heterostructures. Nat. Commun. 2016, 7, 1189410.1038/ncomms11894.

(33) Xu, Z.-Q.; Elbadawi, C.; Tran, T. T.; Kianinia, M.; Li, X.; Liu, D.; Hoffman, T. B.; Nguyen, M.; Kim, S.; Edgar, J. H.; Wu, X.; Song, L.; Ford, M.; Toth, M.; Aharonovich, I. Single Photon Emission from Plasma Treated 2D Hexagonal Boron Nitride. arXiv:1710.07010, 2017. 